\title{
PCB sensor for bacteria detection in saline
}

\author{
Dragana Z. Vasiljevic, Goran M. Stojanovic, Milan R. \\ Radovanovic, Sanja Kojic \\ Faculty of Technical Sciences, University of Novi Sad \\ Novi Sad, Serbia \\ vdragana@uns.ac.rs \\ Bojana Pivas \\ Faculty of Medicine, University of Novi Sad \\ Novi Sad, Serbia
}

\begin{abstract}
This paper represents design, fabrication and characterization of non-invasive capacitive sensor for detection of different concentrations of bacteria such as Pseudomonas aeruginosa and Staphylococcus aureus in saline. The sensor was fabricated in printed circuit board (PCB) technology and it consists of a pair of interdigitated electrodes. In house measurement setup for testing and characterization of sensors has been developed. The characteristics of sensor - the capacitance as a function of frequency for a different concentrations of each bacteria are presented. The obtained results demonstrate different resonant frequencies for each dilution of Pseudomonas aeruginosa and Staphylococcus aureus in physiological saline.
\end{abstract}

Keywords-: capacitive sensor, pseudomonas aeruginosa, staphylococcus aureus, oral health, PCB technology

\section{INTRODUCTION}

Even though 3.5 billion years old, microorganisms are still under-researched, primarily because of its large possibility of changing. They change their properties, increase the virulence and pathogenic potential and because of all this, their recognition and identification is still a big challenge. Bacteria have great potential for division and 24-hour changes 100 generations, and thus gain some new features which are primarily related to their resistance [1]. Pseudomonas aeruginosa is a gram-negative bacillus, strictly aerobic, which is considered a major cause of various infections, such as infections of the wounds after the integrity of the skin or mucous membranes, infections of burns, the urinary and respiratory tract, but this can lead to serious conditions such as bacteremia [2]. Staphylococcus aureus is a gram-positive bacteria, facultative anaerobic, by morphology coca, with characteristic arrangement in the form of clusters or combs. It is an important human pathogen responsible for a variety of diseases, due to its high pathogenic potential [1]. Staphylococcus aureus can cause a wide range of diseases such as local infections, pneumonia, generalized infection and type of bacteremia sepsis [3]. It also has an ability of colonization [1].

\author{
Deana Medic \\ The Institute of Public Health of Vojvodina \\ Faculty of Medicine, University of Novi Sad \\ Novi Sad, Serbia
}

\author{
Roman Sordan \\ L-NESS, Department of Physics, Politecnico di Milano, \\ Como, Italy \\ roman.sordan@polimi.it
}

The most common causes of diseases and infections such as bacteremia, meningitis, serious infections in neonates, hospital infections, endocarditis, nosocomial infections, formation of dental caries, urinary infections, infections of wounds and burns to septicemia, etc. are bacteria [3-6]. Their rapid identification from biological samples is one of the most important goals in human health care. Nowadays, there are two main methods for identification of bacteria presence: (a) the traditional culture associated with biochemical identification, and (b) the polymerase chain reaction. The first one is time consuming, while the second requires specialized personnel training and expensive equipment [7].

Thet et. al. have developed an electrochemical sensor concept for Staphylococcus aureus and Pseudomonas aeruginosa toxin detection using biomimetic lipid vesicles containing potassium ferricyanide [8]. Lentini et. al. presented a novel rapid and cultivation-free method for highly sensitive and rapid detection of Pseudomonas aeruginosa based on the coupling of phage-capture system with optical techniques, namely Fourier Transform Infrared (FTIR) and visible microRaman spectroscopies [9]. The use of modularly designed and site-specifically oriented synthetic antimicrobial peptides as novel recognition agents enabling detection and quantification of Escherichia coli, Pseudomonas aeruginosa, Staphylococcus aureus and Staphylococcus epidermidis has been published in paper[10].

This paper presents interdigitated capacitive (IDC) sensor which is fabricated in cost-effective and easily accessible Printed Circuit Board (PCB) technology. This type of sensor has a high sensitivity for detection of Pseudomonas aeruginosa and Staphylococcus aureus concentration from human isolates. In-house measurement setup tool was developed for testing and characterization purposes. The results show that sensor is able to detect different concentration of Pseudomonas aeruginosa and Staphylococcus aureus in saline.

\section{DESIGN AND FABRICATION OF SENSOR}

The sensor is designed as an IDC composed of eight electrodes with dimensions presented in Fig. 1. The length of each electrode was $6 \mathrm{~mm}$, while the width and the gap were $1.50 \mathrm{~mm}$ and $0.75 \mathrm{~mm}$, respectively. The overall dimension of 
the sensor's design was $21.75 \mathrm{~mm}$ x $9.75 \mathrm{~mm}$.

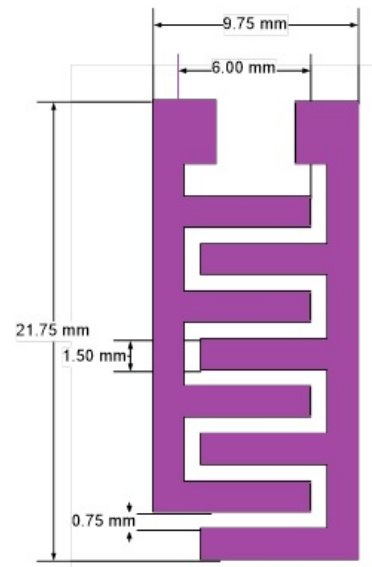

Fig. 1. Schematic of the sensor

The sensor is fabricated in commonly used cost-effective PCB technology. After the fabrication, contacts on sensor were made and sensor was medically sterilized to avoid any possibilities of impurities which could make slightly influence on the measurements.

\section{RESULTS AND DISCUSSION}

Measurements were performed using laboratory measurement setup, shown in Fig. 2. It consists of a sterile test tube filled with different concentration of measured bacteria, test tube holder and HP4194A Impedance/gain-phase analyzer connected to PC. As it can be seen from Fig. 2. sensor is dipped inside of solution in test tube and connected to Impedance analyzer.



Fig. 2. Laboratory measurement setup

As testing material human isolate of Pseudomonas aeruginosa and Staphylococcus aureus from materials which is routinely arrived for treatment in the Centre for Microbiology, Institute of Public Health of Vojvodina is used. From colonies of 24-hour cultures on blood agar (HiMedia, India) for mentioned bacteria, in sterile tubes, suspensions with density $0.5 \mathrm{MCF}$ were made in $4.5 \mathrm{ml}$ of physiological saline using EUCAST standard [11]. Furthermore, this suspension was diluted to the 6 concentrations in range of $10^{-1}$ $\div 10^{-6}$ from initial concentration (Pseudomonas aeruginosa

This work was supported by the Project no. 114-451-2723/2016-01 founded by the Provincial Secretariat for Higher Education and Science of The Province of Vojvodina and partially supported by Ministry of Education, Science and Technological Development within the Project no. TR32016. and Staphylococcus aureus suspensions with density 0.5 MCF in $4.5 \mathrm{ml}$ of physiological saline). For each measurement a new sterile sensor with the same design is used to avoid contamination caused by the previous measurement. All measurements were performed in sterile conditions at the room temperature.

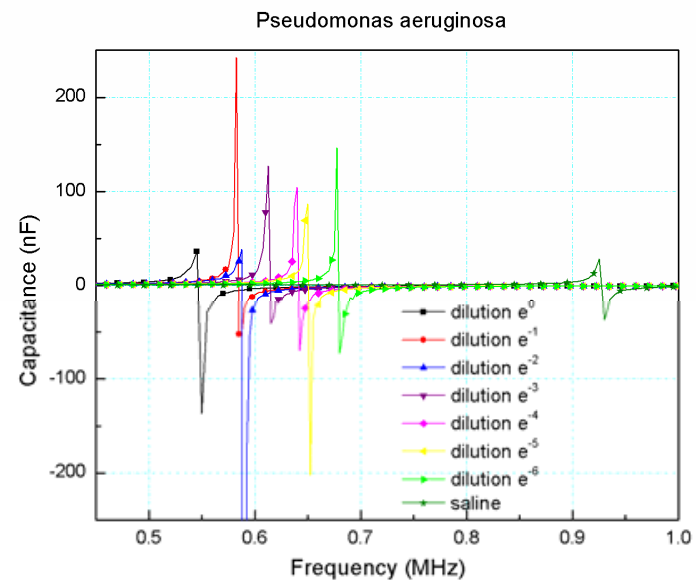

a)

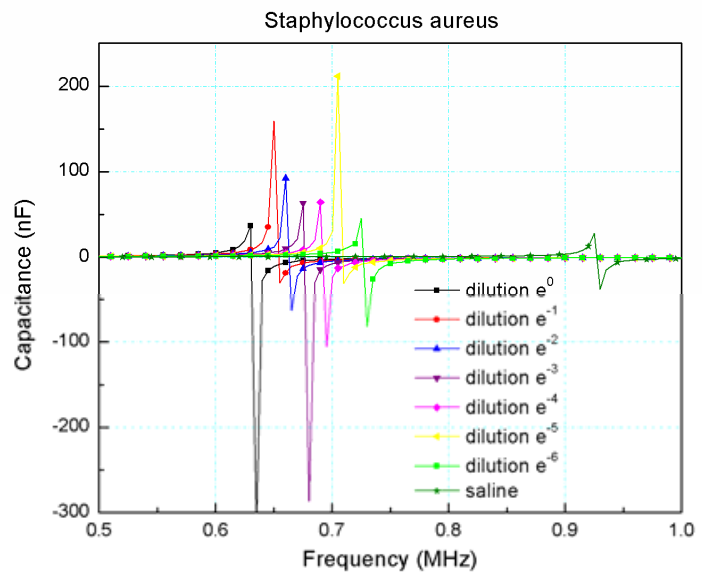

b)

Fig. 3. Capacitance change of the sensor with different concentration of a) Pseudomonas aeruginosa and b) Staphylococcus aureus

Fig. 3. displays the test results of the capacitance versus the frequency for all measured dilutions. It can be seen that each dilution has its own resonant frequency which makes it easily recognizable one from each other. As a reference pure saline was measured, and it has been found that the resonant frequency of saline was $0.9250 \mathrm{MHz}$. In this way the concentration of Pseudomonas aeruginosa and Staphylococcus aureus presence can be easily differentiated and an adequate treatment can be applied. 
Table 1. Resonance frequency of dilutions of Pseudomonas aeruginosa and Staphylococcus aureus in phisiological saline

\begin{tabular}{|c|c|c|}
\hline \multirow{2}{*}{ Dilution } & \multicolumn{2}{|c|}{ Resonance frequency (MHz) } \\
\cline { 2 - 3 } & $\begin{array}{c}\text { Pseudomonas } \\
\text { aeruginosa }\end{array}$ & $\begin{array}{c}\text { Staphylococcus } \\
\text { aureus }\end{array}$ \\
\hline $\mathrm{e}^{0}$ & 0.5446 & 0.6297 \\
\hline $\mathrm{e}^{-1}$ & 0.5827 & 0.6403 \\
\hline $\mathrm{e}^{-2}$ & 0.5874 & 0.6602 \\
\hline $\mathrm{e}^{-3}$ & 0.6128 & 0.6806 \\
\hline $\mathrm{e}^{-4}$ & 0.6397 & 0.6899 \\
\hline $\mathrm{e}^{-5}$ & 0.6507 & 0.7053 \\
\hline $\mathrm{e}^{-6}$ & 0.6784 & 0.7255 \\
\hline
\end{tabular}

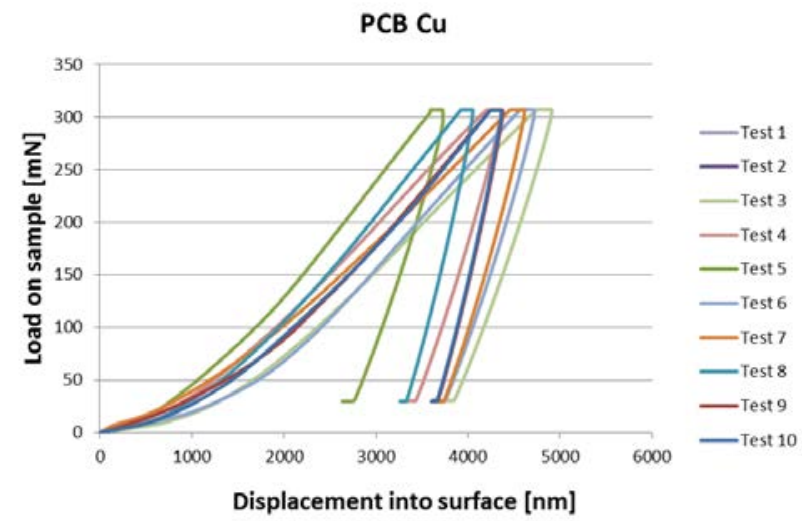

Fig. 4. Applied load as a function of displacement into $\mathrm{Cu}$ conductive layer

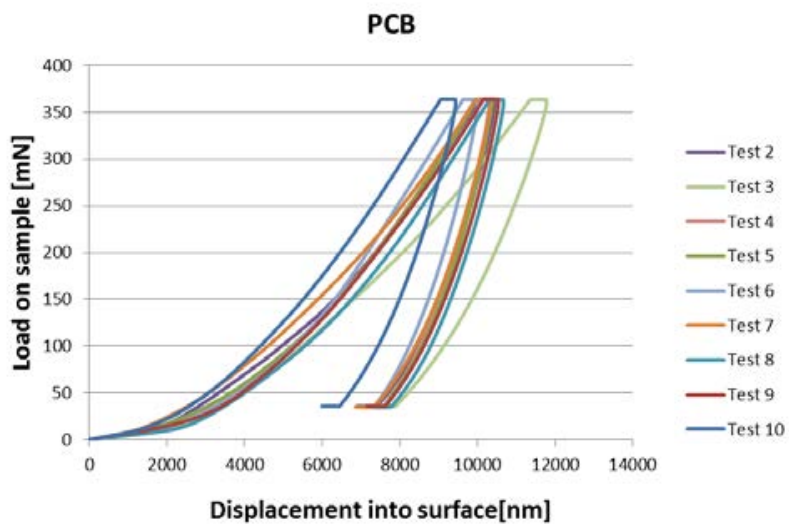

Fig. 5. Applied load as a function of displacement into PCB

As it can be seen from the Table 1. each bacteria dilution has its own resonant frequency value which makes them easily recognizable. Following the values from the Table, it can be seen than resonant frequency of both, diluted Pseudomonas aeruginosa and Staphylococcus aureus increases as the concentration of bacteria in solution decreases. Figures 4 and 5 show mechanical characteristics of proposed sensor using nanoindentation technique. It can be concluded that $\mathrm{Cu}$ part of the sensor has higher hardness than PCB substrate layer.

\section{CONCLUSION}

In this paper capacitive sensor of an IDC design has been fabricated in frequently used and cost-effective PCB technology. The capacitance changes as a function of a frequency were examined by exposing sensors to different concentration of solution of human isolate of Pseudomonas aeruginosa and Staphylococcus aureus. Fabricated sensor design showed an excellent sensitivity to bacteria concentration in physiological saline. The sensor can be used for accurate and fast detection of bacteria in mouth of patients.

\section{REFERENCES}

[1] M. Mihajlović-Ukropina., D. Medić, Z. Jelesić, R. Dautović, Stefan-S. Mikić, S. Sević, "Frequency of methicillin-resistant Staphylococcus aureus strains in clinical specimens obtained from hospitalized patients in 2007”, Med Pregl. 61(Suppl 1), p.p. 27-30, 2008.

[2] A. Japoni, S. Farshad, A. Alborzi, "Pseudomonas aeruginosa: burn infection, treatment and antibacterial resistance”, Iranian Red Crescent Medical Journal, vol. 11, no. 3, pp. 244-253, 2009.

[3] J. Tang, J. Hu, L. Kang, Z. Deng, J. Wu, J. Pan, “The use of vancomycin in the treatment of adult patients with methicillin-resistant Staphylococcus aureus (MRSA) infection: a survey in a tertiary hospital in China”. Int J Clin Exp Med, 8(10), p.p. 19436-19441, 2015.

[4] .S. A. Shelburne, A. M. Chaftari, M. Jamal, I. Al Wohoush, Y. Jiang, S. Abughazaleh, J. Cairo, S. Raad, L. Debiane, I. Raad, "Identification and characterization of catheter-related bloodstream infections due to viridians group streptococci in patients with cancer”, Am J Infect Control, vol. 42, no. 10, pp. 1127-1129, 2014.

[5] X. Chen, Y. Q. Song, H. Y. Xu, B. L. Menghe, H. P. Zhang, Z. H. Sun, "Genetic relationships among Enterococcus faecalis isolates from different sources as revealed by multilocus sequence typing”, J Dairy Sci, vol. 98, no. 8, pp. 5183-5193, 2015.

[6] N. F. Crum-Cianflone, X. Wang, A. Weintrob, T. Lalani, M. Bavaro, J. F. Okulicz, K. Mende, M. Ellis M., B. K. Agan, "Specific behaviors predict Staphylococcus aureus colonization and skin and soft tissue infections among human immunodeficiency virus-infected persons", Open Forum Infect Dis, 2(2), 2015.

[7] M. S. Mannoor, H. Tao, J. D. Clayton, A. Sengupta, D. L. Kaplan, R. R. Naik, N. Verma, F. G. Omenetto, M. C. McAlpine, "Graphene-based wireless bacteria detection on tooth enamel, Nature Communications, vol. 3, no. 763, 2012.

[8] N. T. Thet, A. T. A. Jenkins, "An electrochemical sensor concept for the detection of bacterial virulence factors from Staphylococcus aureus and Pseudomonas aeruginosa”, vol. 59, pp. 104-108, 2015.

[9] G. Lentini, D. Franco, E. Fazio, L.M. De Plano, S. Trusso, S. Carnazza, F. Neri, S.P.P. Guglielmino, "Rapid detection of Pseudomonas aeruginosa by phage-capture system coupled with micro-Raman spectroscopy”, vol. 86, pp. 1-7, 2016.

[10] X. Liu, M. Marrakchi, D. Xua, H. Dong, S. Andreescu, "Biosensors based on modularly designed synthetic peptides for recognition, detection and live/dead differentiation of pathogenic bacteria”, Biosens Bioelectron, 80, p.p. 9-16, 2016.

[11] The European Committee on Antimicrobial Susceptibility Testing (EUCAST). Version 6.0, 2016. 